\title{
Sanguinarine protects against pressure overload-induced cardiac remodeling via inhibition of nuclear factor- $\kappa B$ activation
}

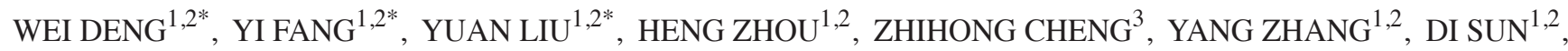

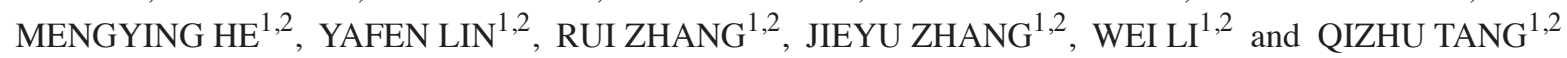 \\ ${ }^{1}$ Department of Cardiology, Renmin Hospital of Wuhan University, Wuhan, Hubei 430060; \\ ${ }^{2}$ Cardiovascular Research Institute of Wuhan University, Wuhan, Hubei 430060; \\ ${ }^{3}$ National Pharmaceutical Engineering Research Center, Shanghai 201203, P.R. China
}

Received September 12, 2013; Accepted March 28, 2014

DOI: $10.3892 / \mathrm{mmr} .2014 .2206$

\begin{abstract}
Cardiac remodeling is a major determinant of heart failure characterized by cardiac hypertrophy and fibrosis. Sanguinarine exerts widespread pharmacological effects, including antitumor and anti-inflammatory responses. In the present study, the effect of sanguinarine on cardiac hypertrophy, fibrosis and heart function was determined using the model induced by aortic banding (AB) in mice. AB surgery and sham surgery were performed on male wild-type C57 mice, aged 8-10 weeks, with or without administration of sanguinarine from one week after surgery for an additional seven weeks. Sanguinarine protected against the cardiac hypertrophy, fibrosis and dysfunction induced by $A B$, as assessed by the heart weight/body weight, lung weight/body weight and heart weight/tibia length ratios, echocardiographic and hemodynamic parameters, histological analysis, and the gene expression levels of hypertrophic and fibrotic markers. The inhibitory effect of sanguinarine on cardiac remodeling was mediated by inhibiting nuclear factor (NF)- $\kappa \mathrm{B}$ signaling pathway activation. The findings indicated that sanguinarine protected against cardiac hypertrophy and fibrosis via inhibiting $\mathrm{NF}-\kappa \mathrm{B}$ activation. These findings may be used to develop a potential therapeutic drug for treating cardiac remodeling and heart failure.
\end{abstract}

\section{Introduction}

Heart failure (HF) is a major health and economic burden worldwide, and its prevalence is continuously increasing (1). The key pathophysiological process that ultimately results

Correspondence to: Professor Qizhu Tang, Department of Cardiology, Renmin Hospital of Wuhan University, 238 Jiefang Road, Wuhan, Hubei 430060, P.R. China

E-mail: qztang@whu.edu.cn

*Contributed equally

Key words: sanguinarine, cardiac remodeling, heart failure, nuclear factor- $\mathrm{\kappa} B$ in heart failure is cardiac remodeling in response to chronic pathological stresses, such as hypertension and myocardial ischemia (2). Cardiac remodeling, which involves myocyte hypertrophy along with interstitial cell proliferation and extracellular matrix remodeling, induces structural and functional changes, mainly in the left ventricle (3). Initially, cardiac remodeling is a beneficial compensatory process, which reduces cardiac wall stress and increases cardiac output, but remodeling ultimately results in the inability of the heart to meet hemodynamic demands. Despite a number of important therapeutic advances in the treatment of symptomatic HF, the prevalence, mortality and cost associated with HF continue to increase (4). Therefore, the development of novel therapeutic strategies to attenuate cardiac remodeling and prevent heart failure is an urgent goal for the biomedical community.

Sanguinarine, derived from the root of Sanguinaria canadensis and other poppy fumaria species, has been demonstrated to exert widespread pharmacological actions, including antimicrobial, antitumor and anti-inflammatory responses (5-8). In particular, sanguinarine was reported to possess cardioprotective outcomes, such as antihypertension, antiplatelet and positive inotropic effects (9). However, the influence of sanguinarine on cardiac remodeling was unknown.

The aim of the present study was to investigate whether sanguinarine improved cardiac hypertrophy and fibrosis in mice.

\section{Materials and methods}

Materials and animal models. Sanguinarine (>98\%) was ordered from Shanghai Winherb Medical S\&T Development Co., Ltd. (Shanghai, China). Adult male C57BL/6 mice, aged 8-10 weeks, were purchased from the Institute of Laboratory Animal Science, Chinese Academy of Medical Sciences (Beijing, China) and acclimated for one week prior to experimental use. The mice were randomly assigned to four groups (Veh-Sham, SAN-Sham, Veh-AB, SAN-AB). Sanguinarine suspensions were freshly prepared for the animal experiments using $0.5 \%$ carboxymethyl cellulose solution. The suspensions were administered to the mice at a constant volume of $1 \mathrm{ml} / 100 \mathrm{~g}$ body weight by oral gavage once a day. The control group was administered the same volume of liquid 
Table I. Echocardiographic and hemodynamic parameters of the effects of sanguinarine on cardiac remodeling induced by $\mathrm{AB}$ in wild-type mice.

\begin{tabular}{lcccc}
\hline Parameter & Veh-Sham & SAN-Sham & Veh-AB & SAN-AB \\
\hline Number & 8 & 8 & 8 & 8 \\
HR (beats/min) & $513 \pm 11$ & $516 \pm 17$ & $517 \pm 9$ & $510 \pm 13$ \\
IVSd (mm) & $0.67 \pm 0.01$ & $0.67 \pm 0.01$ & $0.82 \pm 0.02^{\mathrm{a}}$ & $0.78 \pm 0.01^{\mathrm{a}}$ \\
LVd (mm) & $3.65 \pm 0.03$ & $3.81 \pm 0.04$ & $5.21 \pm 0.02^{\mathrm{a}}$ & $4.56 \pm 0.13^{\mathrm{ac}}$ \\
LVPWd (mm) & $0.67 \pm 0.01$ & $0.68 \pm 0.02$ & $0.83 \pm 0.02^{\mathrm{a}}$ & $0.76 \pm 0.02^{\mathrm{ab}}$ \\
IVSs (mm) & $1.03 \pm 0.02$ & $1.05 \pm 0.02$ & $1.26 \pm 0.03^{\mathrm{a}}$ & $1.13 \pm 0.03^{\mathrm{ac}}$ \\
LVs (mm) & $2.05 \pm 0.03$ & $2.29 \pm 0.05$ & $4.00 \pm 0.13^{\mathrm{a}}$ & $3.19 \pm 0.14^{\mathrm{ac}}$ \\
LVPWs (mm) & $1.04 \pm 0.02$ & $1.06 \pm 0.02$ & $1.24 \pm 0.02^{\mathrm{a}}$ & $1.16 \pm 0.03^{\mathrm{ab}}$ \\
LVEF (\%) & $81.00 \pm 0.76$ & $77.88 \pm 1.11$ & $52.88 \pm 1.33^{\mathrm{a}}$ & $65.00 \pm 1.74^{\mathrm{ac}}$ \\
LVFS (\%) & $43.38 \pm 0.63$ & $41.50 \pm 0.78$ & $23.38 \pm 0.78^{\mathrm{a}}$ & $30.75 \pm 1.22^{\mathrm{ac}}$ \\
ESP (mmHg) & $102.85 \pm 2.38$ & $102.69 \pm 2.29$ & $144.41 \pm 3.04^{\mathrm{a}}$ & $141.11 \pm 8.73^{\mathrm{a}}$ \\
\hline
\end{tabular}

Data represent the mean \pm standard error of the mean. ${ }^{\mathrm{a}} \mathrm{P}<0.01$ vs. the Vehicle-sham group; ${ }^{\mathrm{b}} \mathrm{P}<0.05$ and ${ }^{\mathrm{c}} \mathrm{P}<0.01$ vs. the Vehicle-AB group. Sham, sham surgery; AB, aortic binding; Veh, vehicle; SAN, sanguinarine; HR, heart rate; IVSd, interventricular septal thickness at end-diastole; LVd, left ventricle diastole; LVPWD, left ventricle posterior wall thickness at end-diastole; IVSs, interventricular septal thickness at end-systole; LVs, left ventricle systole; LVPWs, left ventricle posterior wall thickness at end-systole; LVEF, left ventricle ejection fraction; LVFS, left ventricle fractional shortening; ESP, end-systolic pressure.

Table II. General data on the effects of sanguinarine on cardiac remodeling induced by AB in wild-type mice.

\begin{tabular}{lrrrc}
\hline Parameter & Veh-Sham & SAN-Sham & Veh-AB & SAN-AB \\
\hline Number & 10 & 10 & 10 & 10 \\
BW (g) & $28.42 \pm 0.42$ & $28.99 \pm 0.44$ & $29.00 \pm 0.41$ & $28.39 \pm 0.46$ \\
HW (mg) & $122.20 \pm 2.80$ & $120.60 \pm 1.73$ & $236.60 \pm 6.40^{\mathrm{a}}$ & $170.50 \pm 7.79^{\mathrm{ab}}$ \\
LW (mg) & $141.50 \pm 3.25$ & $139.40 \pm 3.19$ & $166.30 \pm 6.04^{\mathrm{a}}$ & $143.40 \pm 3.77^{\mathrm{b}}$ \\
HW/BW (mg/g) & $4.30 \pm 0.08$ & $4.17 \pm 0.08$ & $8.18 \pm 0.27^{\mathrm{a}}$ & $5.99 \pm 0.21^{\mathrm{ab}}$ \\
LW/BW (mg/g) & $4.99 \pm 0.12$ & $4.82 \pm 0.12$ & $5.76 \pm 0.27^{\mathrm{a}}$ & $5.05 \pm 0.09^{\mathrm{b}}$ \\
HW/TL (mg/mm) & $6.66 \pm 0.13$ & $6.43 \pm 0.10$ & $12.65 \pm 0.32^{\mathrm{a}}$ & $9.09 \pm 0.40^{\mathrm{ab}}$ \\
\hline
\end{tabular}

Data represent the mean \pm standard error of the mean. ${ }^{\mathrm{a}} \mathrm{P}<0.01$ vs. the Vehicle-sham group; ${ }^{b} \mathrm{P}<0.01$ vs. the Vehicle-AB group. Sham, sham surgery; AB, aortic banding; SAN, sanguinarine; Veh, vehicle; BW, body weight; HW, heart weight; LW, lung weight; TL, tibia length.

comprised solely of the vehicle solution $(0.5 \%$ carboxymethyl cellulose). Aortic banding (AB) was performed as described previously (10). Treatment with $5 \mathrm{mg} / \mathrm{kg} /$ day sanguinarine or vehicle from one week after AB surgery or sham surgery for seven weeks allowed for critical evaluation. All animal procedures were performed in accordance with the Guide for the Care and Use of Laboratory Animals published by the US National Institutes of Health (NIH publication no. 85-23, revised 1996) and approved by the Institutional Animal Care and Use Committee at Renmin Hospital, Wuhan University (Wuhan, China). All surgeries and subsequent analyses were performed in a blinded manner.

Echocardiographic and hemodynamic evaluation. Cardiac function was determined by transthoracic echocardiography and hemodynamic analysis as described previously (11). Echocardiography was performed in mice anesthetized with
$1.5 \%$ isoflurane, using a MYLAB ${ }^{\mathrm{TM}} 30 \mathrm{CV}$ with a $10-\mathrm{MHz}$ linear-array ultrasound transducer (Esaote S.p.A, Genoa, Italy). The left ventricle (LV) dimensions were assessed in the parasternal short-axis view. End-systole and end-diastole were defined as the phases in which the smallest or largest areas of the LV were obtained, respectively. For hemodynamic measurements, a microtip catheter transducer (SPR-839; Millar Instruments, Houston, TX, USA) was inserted into the right carotid artery and advanced into the left ventricle of mice anesthetized with $1.5 \%$ isoflurane. The signals were continuously recorded using a Millar Pressure-Volume system (MPVS-400; Millar Instruments) and the data were processed by PVAN data analysis software (Millar Instruments).

Histological analysis. Excised hearts from mice which were sacrificed by cervical vertebra dislocation were arrested 
A
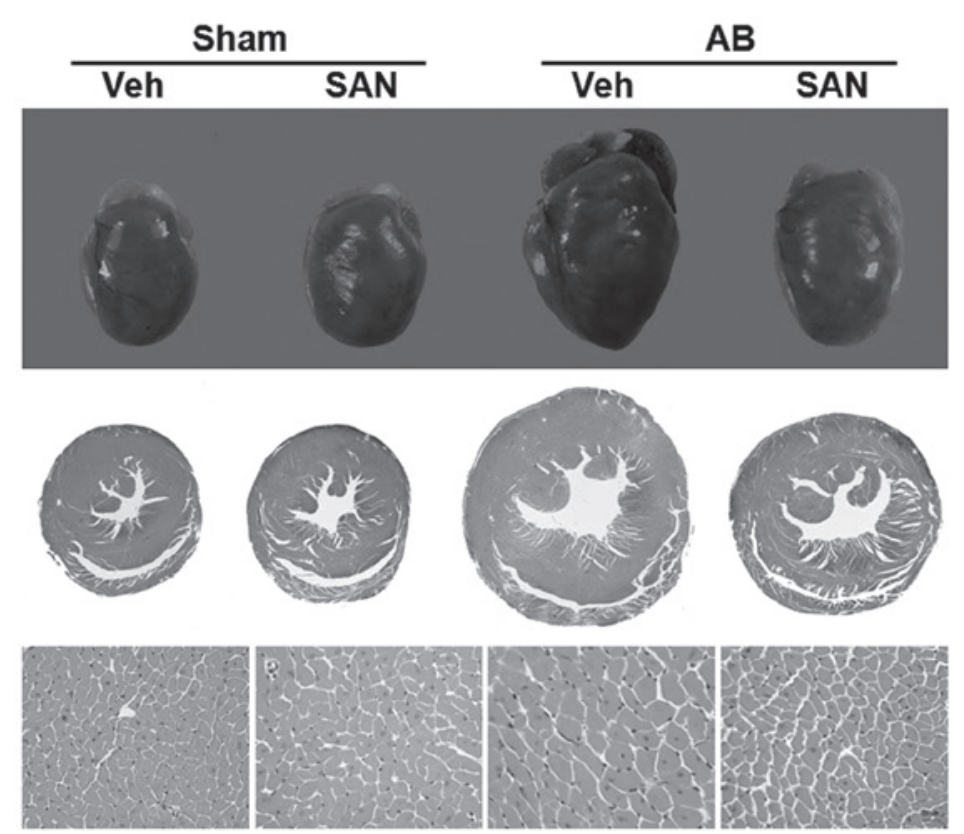

B
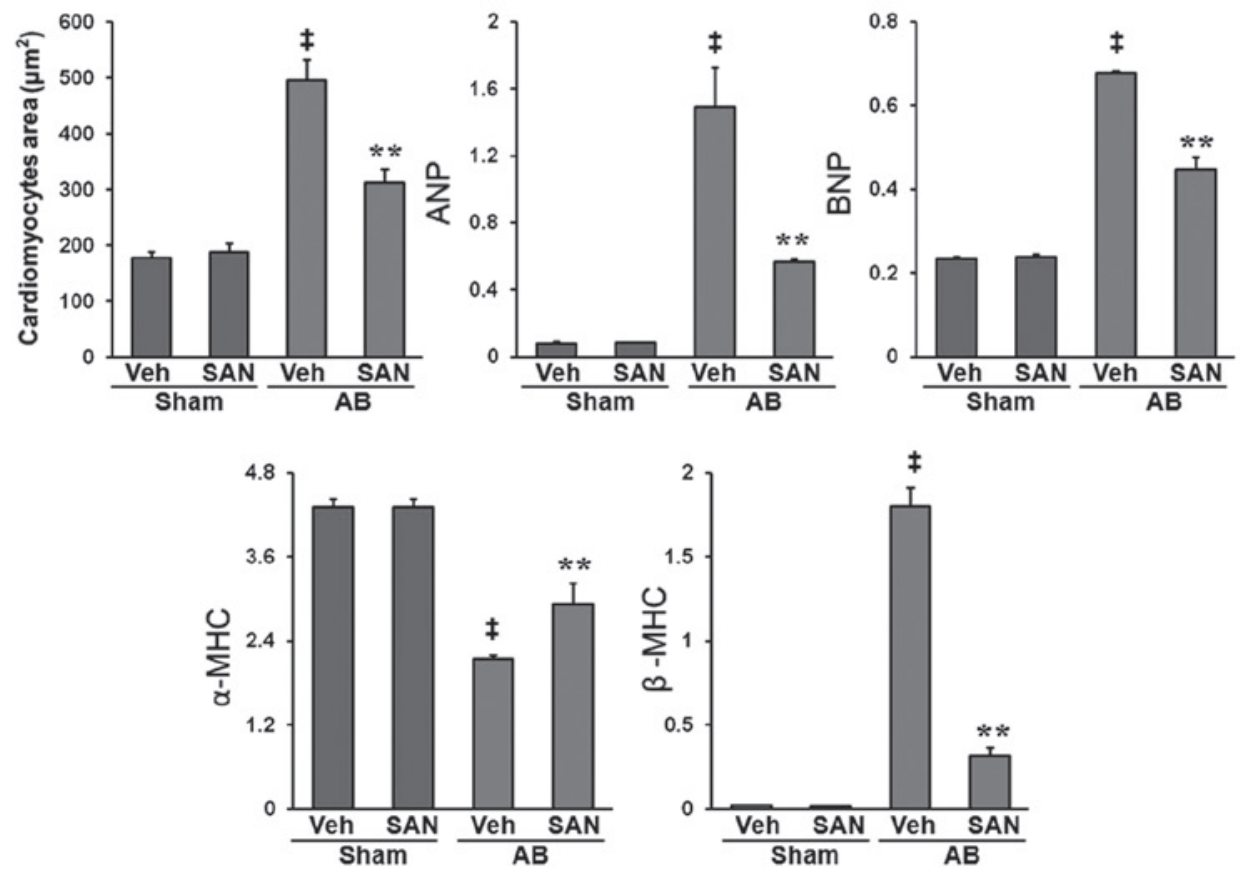

Figure 1. Sanguinarine protects against cardiac hypertrophy induced by pressure overload. (A) Histology results from gross heart observation and hematoxylin-eosin staining after eight weeks of $\mathrm{AB}$ or sham surgery with or without administration of sanguinarine. (B) The myocyte cross-sectional areas and the mRNA expression levels of hypertrophic markers in the left ventricular myocardium after eight weeks of AB with or without administration of sanguinarine $(\mathrm{n}=6)$. Values are presented as the mean \pm standard error of the mean. ${ }^{*} \mathrm{P}<0.01$, compared with the Vehicle-Sham group; ${ }^{* * *} \mathrm{P}<0.01$ compared with the Vehicle-AB group. Sham, sham surgery; AB, aortic banding; SAN, sanguinarine; ANP, atrial natriuretic peptide; BNP, brain natriuretic peptide; MHC, myosin heavy chain.

in diastole with $10 \% \mathrm{KCl}$, weighed, fixed by perfusion with $10 \%$ formalin and embedded in paraffin. The hearts were cut transversely close to the apex to visualize the left and right ventricles. Several sections of each heart (4-5 $\mu \mathrm{m}$ thick) were prepared, stained with hematoxylin and eosin (H\&E) and picrosirius red (PSR) to determine the myocyte cross-sectional area and collagen deposition, and were measured using a quantitative digital image analysis system (Image Pro-Plus, version 6.0; Media Cybernetics, Inc., MD, USA).

Quantitative polymerase chain reaction ( $q P C R$ ). Total RNA was isolated from various organs using TRIzol reagent (Invitrogen Life Technologies, Carlsbad, CA, USA), reverse-transcribed to complementary DNA, and analyzed 
A

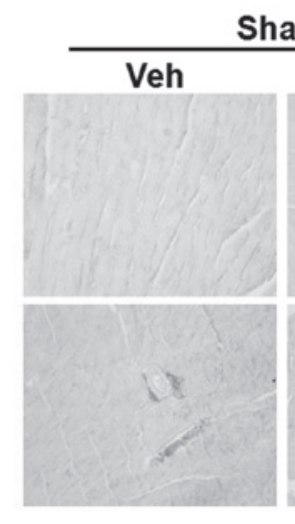

Sham

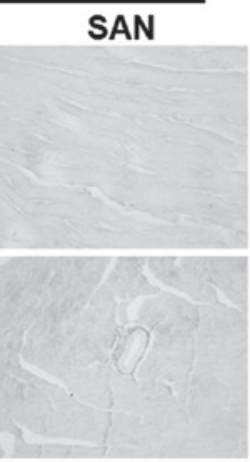

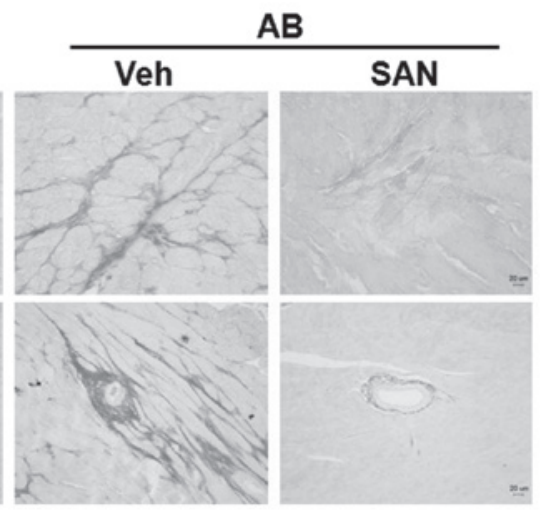

B
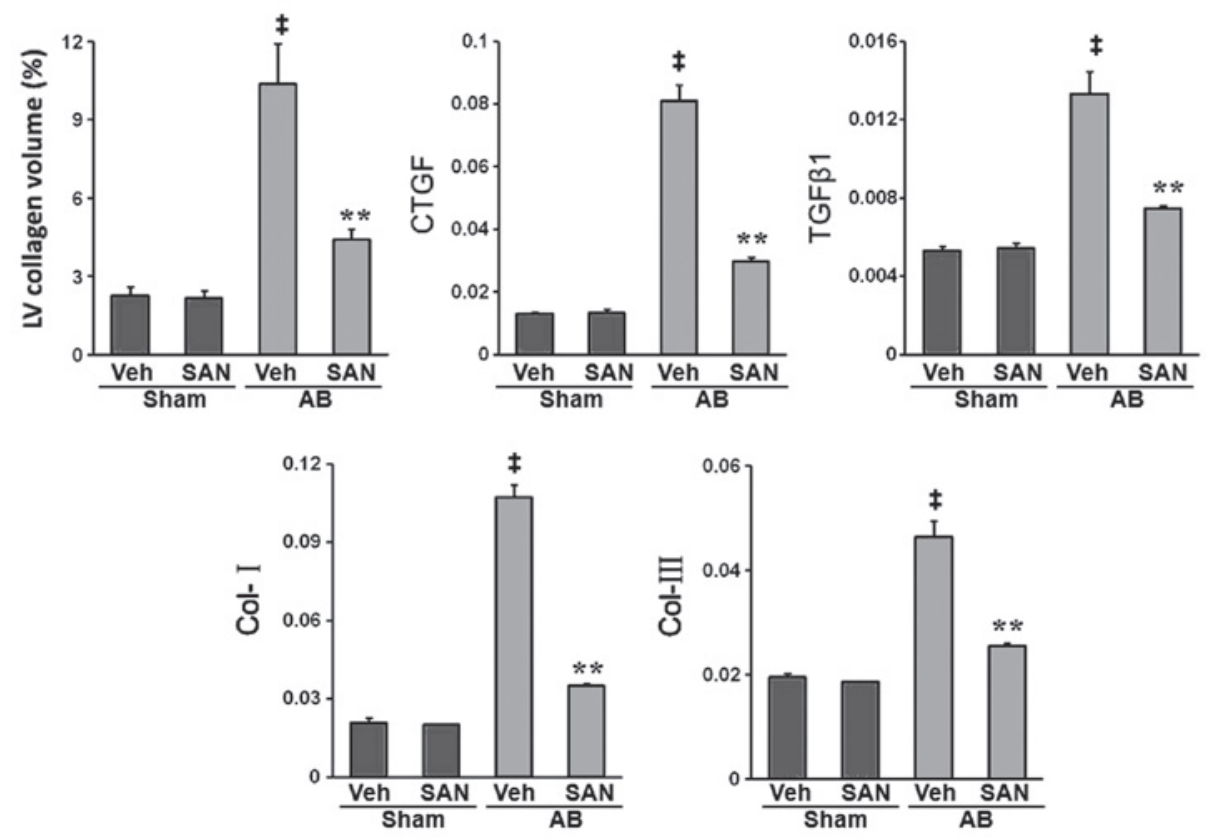

Figure 2. Sanguinarine inhibits cardiac fibrosis induced by pressure overload. (A) Picrosirius red staining of histological sections of the left ventricule was performed for each group after eight weeks of AB. (B) The fibrotic areas in the histological sections and the mRNA expression levels of fibrotic markers in the left ventricular myocardium after eight weeks of $A B$ or sham surgery with or without administration of sanguinarine $(n=6)$. Values are presented as the mean \pm standard error of the mean. ${ }^{*} \mathrm{P}<0.01$, compared with the Vehicle-Sham group; ${ }^{* *} \mathrm{P}<0.01$, compared with the Vehicle-AB group. Sham, sham surgery; $\mathrm{AB}$, aortic banding; SAN, sanguinarine; Veh, vehicle; CTGF, connective tissue growth factor; TGF $\beta 1$, transforming growth factor- $\beta 1$; Col, collagen.

by qPCR using the LightCycler 480 SYBR Green 1 Master mix (Roche Diagnostics, Mannheim, Germany) and the LightCycler 480 qPCR system (Roche Diagnostics). The target gene mRNA expression levels were normalized to those of the internal control GAPDH mRNA and presented relative to the control group.

Western blotting. Protein samples denatured in SDS sample buffer (125 mmol/1 Tris-HCl, pH 6.8, 50\% glycerol, 2\% SDS, $5 \%$ mercaptoethanol and $0.01 \%$ bromophenol blue) were subjected to SDS-PAGE and blotted onto Immobilon-FL transfer membranes (Millipore, Billerica, MA, USA). The membranes were blocked with $5 \%$ skimmed milk in Tris-buffered saline containing $0.1 \%$ Tween-20 (TBST) for $2 \mathrm{~h}$ and were subsequently incubated with primary antibodies against interleukin (IL)-1 $\beta$, IL-6 (R\&D Systems, Minneapolis,

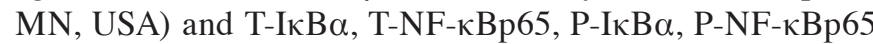
(all from Cell Signaling Technology, Inc., Danvers, MA, USA) overnight at $4^{\circ} \mathrm{C}$. Following three washes in TBST, the membranes were incubated with anti-mouse or anti-rabbit IgG (LI-COR Biosciences, Lincoln, NE, USA) for $1 \mathrm{~h}$. Bands were quantified by the Odyssey infrared imaging system (LI-COR Biosciences) to detect protein expression levels. The specific protein expression levels were normalized to those of a GAPDH control (Santa Cruz Biotechnology, Inc., Santa Cruz, CA, USA) for the total cell lysate.

Statistical analysis. Data are expressed as the mean \pm standard error of the mean. Differences among groups were determined by two-way analysis of variance followed by Tukey's post hoc test. Comparisons between two groups were performed using an unpaired Student's t-test. $\mathrm{P}<0.05$ was considered to indicate a statistically significant difference.

\section{Results}

Sanguinarine protects against cardiac hypertrophy induced by pressure overload. To investigate the effects of sanguinarine 
A

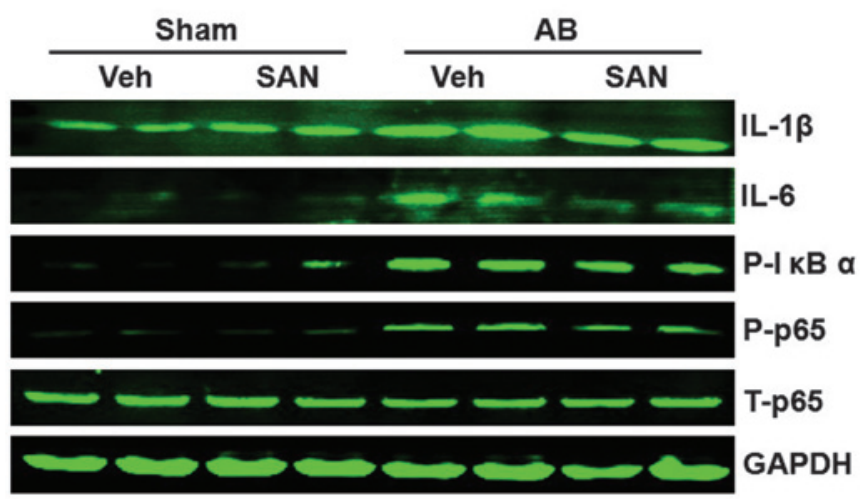

$\mathbf{B}$

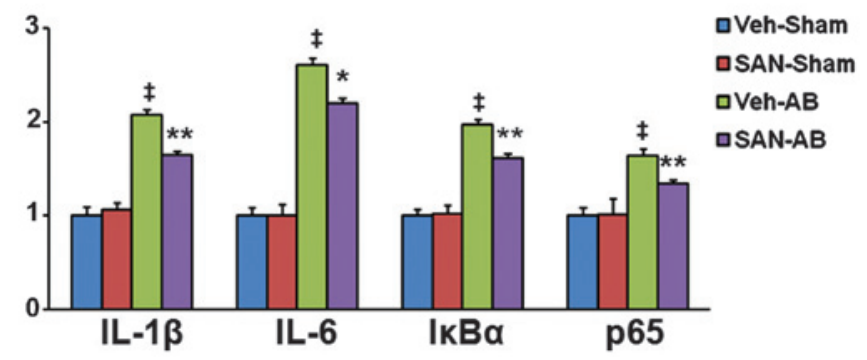

Figure 3. Reversal effect of sanguinarine on the myocardial NF- $\mathrm{kB}$ signaling pathway in response to pressure overload. (A) Phosphorylation of proteins associ-

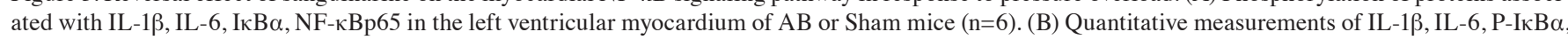
$\mathrm{P}-\mathrm{NF}-\kappa \mathrm{K}$ 65 5 protein relative to GAPDH. Values are presented as the mean \pm standard error of the mean. ${ }^{\star} \mathrm{P}<0.01$ compared with the Vehicle-Sham group;

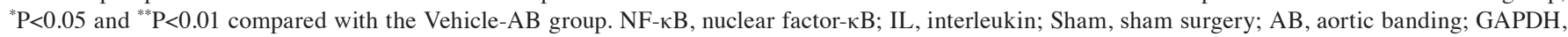
glyceraldehyde 3-phosphate dehydrogenase; Veh, vehicle; SAN, sanguinarine.

on cardiac hypertrophy, all wild-type mice were subjected to AB surgery or sham surgery with or without oral sanguinarine administration from one week after surgery.

In the present study, sanguinarine inhibited the development of cardiac hypertrophy after eight weeks of AB. The beneficial effects of sanguinarine treatment were indicated by significant increases in LV ejection fraction and LV fractional shortening, and significant reductions in LV diastole, LV systole, interventricular septal thickness at end-diastole, interventricular septal thickness at end-systole, LV posterior wall thickness at end-diastole, LV posterior wall thickness at end-systole, and the heart weight (HW)/body weight (BW), lung weight/BW and $\mathrm{HW} /$ tibia length ratios compared with the vehicle AB group (Tables I and II). Gross heart and H\&E staining indicated that the mice that received oral administration of sanguinarine exhibited markedly reduced cardiac hypertrophy and myocyte cross-sectional areas (Fig. 1A). The expression levels of AB-mediated hypertrophic markers, including atrial natriuretic peptide, brain natriuretic protein and $\beta$-myosin heavy chain $(\beta-\mathrm{MHC})$ were significantly reduced in the mice treated with sanguinarine whereas those of $\alpha$-MHC were increased $(\mathrm{P}<0.01$; Fig. $1 \mathrm{~B})$ compared with the expression levels in the Vehicle-AB mice. These results suggest that sanguinarine negatively regulated the extent of the cardiac hypertrophy and dysfunction induced by pressure overload.

Sanguinarine inhibits cardiac fibrosis induced by pressure overload. To determine the extent of fibrosis in the heart, paraffin-embedded sections were stained with PSR. Marked perivascular and interstitial fibrosis was detected using PSR staining in vehicle-fed and sanguinarine-fed mice that were subjected to AB (Fig. 2A). However, the extent of cardiac fibrosis and the LV collagen volume fraction were markedly reduced in sanguinarine-fed mice (Fig. 2A). Subsequent analysis of the mRNA expression levels of fibrotic mediators, such as transforming growth factor- $\beta 1$ (TGF $\beta 1$ ), connective tissue growth factor (CTGF), collagen I and collagen II, demonstrated a reduced response to fibrosis in sanguinarine-fed mice (Fig. 2B). These results suggest that sanguinarine inhibited the cardiac fibrosis induced by pressure overload.

Sanguinarine inhibits myocardial nuclear factor $(N F)-\kappa B$ activation in response to pressure overload. To investigate the molecular mechanisms by which sanguinarine inhibits cardiac remodeling, the effects of sanguinarine on the $\mathrm{NF}-\kappa \mathrm{B}$ signaling pathway were examined. As expected, AB induced a significant increase in expression levels of IL-1 $\beta$, IL-6, $\mathrm{I} \kappa \mathrm{B} \alpha$ and $\mathrm{NF}-\kappa \mathrm{B}$ p 65 , in the hearts of the vehicle-fed mice in comparison with those receiving sham surgery (Fig. 3A and B). However, the pressure overload-induced activation was markedly inhibited in the hearts of the sanguinarine-fed mice (Fig. 3A and B).

\section{Discussion}

Progress has been achieved in understanding the molecular and cellular processes of heart failure, but this disease remains a predominant cause of illness and mortality in the aging society. Novel treatments that target disease mechanisms at the cellular and whole-organ level are required to halt and reverse the consequences of cardiac remodeling, which include cardiac hypertrophy and fibrosis. The present study used a mechanical overload-induced cardiac remodeling 
paradigm. The main findings were as follows: Sanguinarine inhibited the cardiac hypertrophy, fibrosis and dysfunction induced by pressure overload; sanguinarine blocked the myocardial $\mathrm{NF}-\kappa \mathrm{B}$ signaling pathway and inflammatory activation in response to pressure overload. Thus, to the best of our knowledge sanguinarine was shown for the first time, to effectively protect against cardiac remodeling and dysfunction.

Several studies have observed that sanguinarine inhibited the $\mathrm{NF}-\kappa \mathrm{B}$ signaling pathway and inflammation to execute its biological effects $(8,12)$. Chronic inflammation is a hallmark of HF, and inflammatory mediators are important in processes within cardiac remodeling, including cardiomyocyte hypertrophy, alterations in fetal gene expression levels and interstitial fibrosis $(13,14)$. Inhibition of the $\mathrm{NF}-\kappa \mathrm{B}$ signaling pathway ameliorates chronic infusion of angiotensin II (AngII) or pressure overload-induced myocardial inflammation and cardiac hypertrophy $(15,16)$. Pressure overload promotes cardiac fibroblast proliferation and extracellular matrix accumulation, thereby exacerbating cardiac fibrosis and subsequent heart failure. NF- $\kappa \mathrm{B}$ inhibition may suppress pressure overload or AngII-induced CTGF expression and TGF $\beta$-Smad signaling pathway activation, to decrease the extent of cardiac fibrosis and improve heart function $(14,17)$. In the present study, sanguinarine was identified to attenuate cardiac remodeling via inhibiting the activation of $\mathrm{NF}-\kappa \mathrm{B}$ and downstream pro-inflammatory cytokines, including IL-1 $\beta$ and IL-6.

To the best of our knowledge, this demonstrates for the first time that sanguinarine is effective in inhibiting cardiac remodeling and preserving heart function in banding mice. In addition, these results provide experimental evidence for the application of sanguinarine in the treatment of cardiac remodeling and heart failure.

\section{Acknowledgements}

This study was supported by the National Nature Science Foundation of China (grant nos. 81270303 and 81300104) and the Planning Project of Innovative Experiment of Wuhan University's Undergraduate (grant nos. S2013746).

\section{References}

1. Shah AM and Mann DL: In search of new therapeutic targets and strategies for heart failure: recent advances in basic science. Lancet 378: 704-712, 2011.
2. Gjesdal O, Bluemke DA and Lima JA: Cardiac remodeling at the population level - risk factors, screening, and outcomes. Nat Rev Cardiol 8: 673-685, 2011.

3. Barry SP and Townsend PA: What causes a broken heart - molecular insights into heart failure. Int Rev Cell Mol Biol 284: 113-179, 2010

4. Houser SR, Margulies KB, Murphy AM, Spinale FG, Francis GS, Prabhu SD, Rockman HA, Kass DA, Molkentin JD, Sussman MA and Koch WJ; American Heart Association Council on Basic Cardiovascular Sciences, Council on Clinical Cardiology and Council on Functional Genomics and Translational Biology: Animal models of heart failure: a scientific statement from the American Heart Association. Circ Res 111: 131-150, 2012.

5. Adhami VM, Aziz MH, Mukhtar H and Ahmad N: Activation of prodeath Bcl-2 family proteins and mitochondrial apoptosis pathway by sanguinarine in immortalized human $\mathrm{HaCaT}$ keratinocytes. Clin Cancer Res 9: 3176-3182, 2003.

6. Dinkova-Kostova AT: Phytochemicals as protectors against ultraviolet radiation: versatility of effects and mechanisms. Planta Med 74: 1548-1559, 2008.

7. De Stefano I, Raspaglio G, Zannoni GF, Travaglia D, Prisco MG, Mosca M, Ferlini C, Scambia G and Gallo D: Antiproliferative and antiangiogenic effects of the benzophenanthridine alkaloid sanguinarine in melanoma. Biochem Pharmacol 78: 1374-1381, 2009.

8. Niu X, Fan T, Li W, Huang H, Zhang Y and Xing W: Protective effect of sanguinarine against acetic acid-induced ulcerative colitis in mice. Toxicol Appl Pharmacol 267: 256-265, 2013.

9. Mackraj I, Govender T and Gathiram P: Sanguinarine. Cardiovasc Ther 26: 75-83, 2008.

10. Zong J, Deng W, Zhou H, Bian ZY, Dai J, Yuan Y, Zhang JY, Zhang R, Zhang Y, Wu QQ, et al: 3,3'-Diindolylmethane protects against cardiac hypertrophy via 5'-adenosine monophosphate-activated protein kinase-alpha2. PLoS One 8: e53427, 2013.

11. Deng W, Zong J, Bian Z, Zhou H, Yuan Y, Zhang R, Guo H, Zhang Y, Shen D, Li H and Tang Q: Indole-3-carbinol protects against pressure overload induced cardiac remodeling via activating AMPK-alpha. Mol Nutr Food Res 57: 1680-1687, 2013

12. Pěnčiková K, Kollár P, Müller Závalová V, Táborská E, Urbanová $\mathrm{J}$ and Hošek J: Investigation of sanguinarine and chelerythrine effects on LPS-induced inflammatory gene expression in THP-1 cell line. Phytomedicine 19: 890-895, 2012.

13. Dai J, Shen DF, Bian ZY, Zhou H, Gan HW, Zong J, Deng W, Yuan Y, Li F, Wu QQ, et al: IKKi deficiency promotes pressure overload-induced cardiac hypertrophy and fibrosis. PLoS One 8: e53412, 2013

14. Ma Y, Chen Y, Yang Y, Chen B, Liu D, Xiong Z, Zhang C and Dong Y: Proteasome inhibition attenuates heart failure during the late stages of pressure overload through alterations in collagen expression. Biochem Pharmacol 85: 223-233, 2013.

15. Kawano S, Kubota T, Monden Y, Kawamura N, Tsutsui H, Takeshita A and Sunagawa K: Blockade of NF-kappaB ameliorates myocardial hypertrophy in response to chronic infusion of angiotensin II. Cardiovasc Res 67: 689-698, 2005

16. Wu S, Yin R, Ernest R, Li Y, Zhelyabovska O, Luo J, Yang Y and Yang Q: Liver X receptors are negative regulators of cardiac hypertrophy via suppressing NF-kappaB signalling. Cardiovasc Res 84: 119-126, 2009.

17. Cai Y, Yu SS, Chen TT, Gao S, Geng B, Yu Y, Ye JT and Liu PQ: EGCG inhibits CTGF expression via blocking NF-kappaB activation in cardiac fibroblast. Phytomedicine 20: 106-113, 2013. 